\title{
Cultural differences in the misperception of exponential growth
}

\author{
GIDEON KEREN \\ Institute for Perception TNO, Soesterberg, The Netherlands
}

\begin{abstract}
Previous studies have demonstrated that exponential growth functions are grossly underestimated by human subjects. The extent to which this underestimation depends on past experience was investigated in the present study, in which subjects were required to estimate future prices of a product based on a given rate of inflation. The subjects were drawn from two different populations (Canada and Israel) which had been exposed to radically different rates of inflation during the last few years. Underestimation of the growth function (i.e., the inflation rate) was observed for all subjects, but there was a significant (not just in the statistical sense) difference between subjects, depending on the population from which they were drawn. The magnitude of underestimation for Israeli subjects was significantly smaller, a result that was confirmed in an additional experiment (after the change in the Israeli monetary unit). The results are discussed within the framework of subjective scales of numbers.
\end{abstract}

In several recent papers, Wagenaar and his coworkers (Timmers \& Wagenaar, 1977; Wagenaar \& Sagaria, 1975; Wagenaar \& Timmers, 1978, 1979) have shown that exponential growth presented by numerical series, graphs, or in a direct nonnumerical diagrammatic form (e.g., presentation of duckweed multiplying itself in a pond) is misperceived and grossly underestimated by human subjects. Wagenaar and his colleagues have replicated the finding under various conditions (manipulating different variables), thus suggesting that the phenomenon is rather robust. The underestimation bias was less severe for descending than for ascending series, and people tended to provide somewhat more accurate responses when they observed fewer data points. On the other hand, "Neither mathematical sophistication of the subjects nor experience with growth processes changed this effect"' (Wagenaar \& Timmers, 1979, p. 240).

Recently, Hogarth (Note 1) has claimed that several reports on systematic dysfunctional consequences of judgmental heuristics (e.g., the heuristics described by Tversky \& Kahneman, 1974) have not specified the conditions in which such heuristics are applied. Hogarth's major claim was that, although real-life judgments are by and large part of a continuous, interactive process between the person and his environment, most of the laboratory studies-including those that indicated deficiencies in the extrapolation of exponential growth patterns-have focussed on dis-

The author would like to acknowledge the help of M. Bar Hillel, G. Ben Shaker, and R. Falk in collecting data from Israeli subjects, and to W. A. Wagenaar and an anonymous reviewer for helpful comments. Requests for reprints should be sent to Gideon Keren, Institute for Perception TNO, Kampweg 5, 3769 DE Soesterberg, The Netherlands. crete incidents. Hogarth questions the ecological validity of these studies because the implicit assumptions and optimal strategies under discrete static conditions are not necessarily identical to those under a dynamic environment. In particular, Hogarth (Note 1) conducted an experiment in which one group was tested under static conditions (a replication of Wagenaar \& Sagaria, 1975) and the other group was tested under dynamic conditions with continuous feedback. As predicted, the second group's performance was much more accurate than that of the first,' and responses were very close to the correct values.

Hogarth's assertion regarding the ecological validity of static conditions is only partly justified. Although man is usually acting in a dynamic and changing environment that often provides feedback, he may nevertheless confront situations in which exponential growth has to be estimated in conditions similar to the static ones used by Wagenaar and his co-workers. Hence, static conditions should not be considered a priori as ecologically invalid. In that respect, it should also be noted that ecological validity varies on a continuum rather than in an all-or-none fashion.

In addition to the dynamic-static classification, there are other dimensions relevant to considerations of ecological validity. From a functional viewpoint, and the assumption of limited capacity, it follows that the organism will develop strategies and heuristics that are adaptive to his environment. In the case of estimating growth functions, it is apparent that the subject must employ some heuristics or intuitive devices, since he is unable to carry out the rather complicated calculations required by the exponential growth function (even if he/she were to know the exact function). Although the exact nature of such an alleged heuristic is not known, we may rule out cer- 
tain hypotheses. For instance, it may be tempting to suggest that subjects use a linear rather than an exponential function. The results of Wagenaar and his colleagues rule out such an interpretation, since the shape of the functions produced by their subjects was clearly nonlinear and in fact resembled very much the pattern of a power function. The locus of the underestimation seems to be the carrying out of the power operation rather than the use of a wrong rule (e.g., a linear equation). It is plausible that different magnitudes of growth (i.e., different power values) will induce different values for the parameters of a given heuristic strategy. It is also possible that the scale used by the subject is not fixed: As Luce (1972) has pointed out, the measuring devices of the organism are flexible and may change ${ }^{2}$ in order to adapt to environmental conditions.

The major purpose of the present investigation was to test the extent to which environmental conditions (and previous experience) might affect the perception and accuracy of estimates of exponential growth functions. We looked, therefore, for two populations of subjects with exposures to different growth rates, who might consequently have developed different strategies and scales for estimating exponential growth. We used a task in which subjects were asked to predict the future price of a given product based on different rates of inflation. Subjects were recruited from two populations, which have in the last few years experienced rather different rates of inflation. In the first experiment, we used Canadian subjects who had experienced an inflation rate of $10 \%-15 \%$ in the previous few years; in the second experiment, we employed students from Israel, a country in which the rate of inflation during the previous 3 years had been in the range of $100 \%-150 \%$.

\section{EXPERIMENT 1}

\section{Method}

Design. A $2 \times 2$ between-subjects design was employed in which each subject participated in one of the four conditions. The subjects had to estimate (forecast) the future price of a given product. The two variables manipulated between subjects were the initial (current) price of the product-either a high- or a low-priced item ( $\$ 3$ and $\$ 1,500$ or $\$ 3$ and $\$ 1,000$ )-and an assumed fixed rate of inflation in the economy which was either high $(70 \%)$ or low $(13 \%)$.

Procedure. The subjects were tested in small class groups (20 to 30 subjects each) and randomly assigned to one of the four conditions. The instructions and task were typed on a single page, and the subjects were given $75 \mathrm{sec}$ to read the page and respond. This relatively short time was employed in all the three experiments reported here, and was strictly enforced in order to avoid any actual calculation. The instructions emphasized the intuitive nature of the task. The following is an example of a questionnaire for one of the conditions (low initial price, low rate of inflation):

"The rate of inflation for food items (according to statistical sources in Canada) during the last few years has actually been $13 \%$. For instance, the average price for a pound of high quality steak during the last 4 years has been:
$1977 \$ 2.08$
$1978 \$ 2.35$
$1979 \$ 2.65$
$1980 \$ 3.00$

which represents an increase of $13 \%$ each year. Assuming that this same rate of inflation will continue for the next thirteen years, what is your best estimate for the price of a pound of high quality steak in thirteen years, i.e., in 1993. Please give your answer in $\$$ and cents. ${ }^{3}$

The subjects were initially instructed about the nature of the task and were told that they should avoid written calculations, since we were interested in their immediate, intuitive estimates right after they finished reading the question. The 75-sec time constraint was used to assure that the subjects would have insufficient time to carry out exact calculations.

Subjects. The subjects were undergraduate students at the University of Toronto, and were recruited from classes that did not exceed 30 students. Testing took place at the beginning of the class meeting preceding the lecture.

\section{Results}

The results are given in the left-hand side of Table 1 . The first two rows define the condition in terms of the current price of the product $\left(\mathrm{Y}_{0}\right)$ and the given rate of inflation $(p)$. The third row $\left(Y_{13}\right)$ provides the normatively correct predictions given by

$$
Y_{n}=Y_{o}\left(1+\frac{p}{100}\right)^{n}
$$

where $Y_{0}$ is the initial price, $Y_{n}$ is the predicted price after $n$ years, and $p$ is the assumed inflation rate. med $\hat{Y}_{13}$ is the median response of subjects in a given group. The value of $\hat{p}$ is an estimate of the rate of inflation necessary to account for the subjects' responses if the normative rule were employed (i.e., solving for $p$, given $\left.\hat{Y}_{13}, Y_{o}, n\right)$. Similarly, $\hat{n}$ is an estimate of the number of years necessary to account for the subjects' responses if the normative rule were employed (i.e., solving for $n$, given $\hat{Y}_{13}, Y_{0}, p$ ).

The results show that our subjects exhibit a marked underestimation of the exponential function under all four conditions. The theoretical model described later in the discussion suggests that the manipulation of the initial price and the rate of inflation have little effect on the magnitude of underestimation, which is congruent with previous reports of Wagenaar and his co-workers. This point will be elaborated below.

\section{EXPERIMENT 2}

The second experiment was almost identical to Experiment 1 , except that the subjects were Israeli undergraduate students from the Hebrew University of Jerusalem. In addition, the prices were expressed in Israeli pounds (at that time the exchange rate was approximately 60 pounds to one Canadian dollar) and the initial prices of products were somewhat different (25 and 350 pounds) (see Table 1). Except for the 
Table 1

Summary of Results for the Three Experiments*

\begin{tabular}{|c|c|c|c|c|c|c|c|c|c|c|c|c|}
\hline \multirow[b]{2}{*}{$\begin{array}{c}Y_{0} \\
p\end{array}$} & \multicolumn{4}{|c|}{ Experiment 1} & \multicolumn{4}{|c|}{ Experiment 2} & \multicolumn{4}{|c|}{ Experiment 3} \\
\hline & $\begin{array}{c}3 \\
13 \\
\end{array}$ & $\begin{array}{c}1500 \\
13 \\
\end{array}$ & $\begin{array}{c}3 \\
70 \\
\end{array}$ & $\begin{array}{c}1000 \\
70 \\
\end{array}$ & $\begin{array}{l}25 \\
13 \\
\end{array}$ & $\begin{array}{c}350 \\
13 \\
\end{array}$ & $\begin{array}{l}25 \\
70 \\
\end{array}$ & $\begin{array}{c}350 \\
70 \\
\end{array}$ & $\begin{array}{l}2,5 \\
13 \\
\end{array}$ & $\begin{array}{l}35 \\
13 \\
\end{array}$ & $\begin{array}{l}2,5 \\
70 \\
\end{array}$ & $\begin{array}{l}35 \\
70 \\
\end{array}$ \\
\hline $\begin{array}{l}\mathrm{Y}_{13} \hat{\mathrm{H}} \\
\text { med } \hat{\mathrm{Y}}_{13} \\
\hat{\mathrm{p}} \\
\hat{\mathrm{n}} \\
\mathrm{N}\end{array}$ & $\begin{array}{l}14.7 \\
7.7 \\
7.50 \\
7.64 \\
45\end{array}$ & $\begin{array}{l}7347.0 \\
4500 \\
8.85 \\
8.96 \\
45\end{array}$ & $\begin{array}{l}2971.4 \\
20.7 \\
16.10 \\
3.65 \\
42\end{array}$ & $\begin{array}{l}990457.8 \\
13500 \\
22.20 \\
4.90 \\
41\end{array}$ & $\begin{array}{l}122.4 \\
106.4 \\
11.75 \\
11.86 \\
48\end{array}$ & $\begin{array}{l}1714.3 \\
1485 \\
11.76 \\
11.84 \\
37\end{array}$ & $\begin{array}{l}24761 \\
3000 \\
44.50 \\
9.02 \\
39\end{array}$ & $\begin{array}{l}346660 \\
22500 \\
37.80 \\
7.85 \\
35\end{array}$ & $\begin{array}{l}12.2 \\
9.5 \\
10.75 \\
10.95 \\
36\end{array}$ & $\begin{array}{l}171.4 \\
128.5 \\
10.55 \\
10.65 \\
32\end{array}$ & $\begin{array}{l}2476 \\
352 \\
46.40 \\
9.35 \\
35\end{array}$ & $\begin{array}{l}34666 \\
2350 \\
38.20 \\
7.95 \\
38\end{array}$ \\
\hline
\end{tabular}

Note $-Y_{0}=$ initial (current) price: $p=$ inflation rate in percent: $Y_{13}=$ predicted price after 13 years according to the normative rule (Equation 1); med $\hat{Y}_{13}=$ median predicted price after 13 years based on subjects' responses; $\dot{p}=$ estimated rate of inflation based on subjects' responses in percent; $\hat{n}=$ estimated number of years based on subjects' responses; $N=$ number of subjects. ${ }^{*}$ Note that $Y_{0}, Y_{1_{3}}$, and $\hat{Y}_{1_{3}}$ are expressed in Canadian dollars for the Canadian subjects and in Israeli pounds for Israeli subjects. At the time the first two studies were conducted the approximate rate of exchange was $\$ 1=60$ pounds. The rate of exchange during the conduct of Experiment 3 was approximately $\$ 1=12$ shekels.

above modifications, an effort was made to replicate Experiment 1 as closely as possible.

\section{Results}

The results of Experiment 2 are summarized in the middle columns of Table 1. Again, misperception of growth is evident, although the extent of underestimation is much smaller than it was in the Canadian population. This is particularly noticeable for the $13 \%$ inflation conditions where the median response of the Israeli subjects is quite close to the normatively correct prediction.

From inspecting Table 1 it is evident that the median predictions of the Israeli population are far more accurate than those of the Canadian population, although both groups exhibit a tendency toward underestimation. Of particular interest, however, is the nature of the difference in the predictions of subjects from the two populations. This is discussed in the following section.

\section{Discussion}

Timmers and Wagenaar (1977) and Wagenaar and Sagaria (1975) have proposed a model to account for subjects' misperception of exponential growth. According to this model, subjects underestimate the exponent of the function by a factor $\beta$, and attempt to "correct" for it (insufficiently, of course) by multiplying by a factor $\alpha$ which is independent of the rate of growth. Thus, to account for the observed responses, we may modify the normative equation (1) as follows:

$$
\hat{\mathbf{Y}}_{\mathrm{n}}=\alpha \mathrm{Y}_{\mathrm{o}}(1+\mathrm{p})^{\beta \mathrm{n}}
$$

or, alternatively, in logarithmic form

$$
\ln \hat{Y}_{n}=\ln \alpha+\ln Y_{o}+\beta n \ln (1+p) \text {. }
$$

Equations 2 and 3 both represent a form of the descriptive model proposed by Wagenaar and his coworkers.

Using Equation 3 and carrying out a separate analysis for Canadian and Israeli subjects, we estimated the values of $\alpha$ and $\beta$ for subjects in the small and large initial price groups, separately. For each condition of initial price (high or low) and for each population, we had responses for two different rates of inflation, thus enabling us to construct two equations to solve for $\alpha$ and $\beta$. The assumption here, as well as in Figure 1, is that $\alpha$ and $\beta$ are invariant with respect to other possible rates of inflation (in the range of $13 \%-70 \%$ ). The results are summarized in Table 2. As can be seen, the difference between the groups that initially received a small price and those that initially received a large price is rather moderate and in opposite directions for the two populations. The significant (not necessarily in the statistical sense) result is the difference in magnitude of the $\beta s$ between the two populations. The estimated $\beta$ s for the Canadian population are .18 and .26 for the initially small and initially large price, respectively, and are of the same magnitude as the $\beta$ s reported by Timmers and Wagenaar (1977) and Wagenaar and Sagaria (1975). In contrast, the magnitude of the estimated $\beta$ s for Israeli subjects is more than doubled,

Table 2

Estimated Values of $\alpha$ and $\beta$ as a Function of Population and Initial Price

\begin{tabular}{clccc}
\hline \multirow{2}{*}{ Experiment } & Population & $\begin{array}{c}\text { Initial } \\
\text { Price }\end{array}$ & $\begin{array}{c}\text { Estimated } \\
\alpha\end{array}$ & $\begin{array}{c}\text { Estimated } \\
\beta\end{array}$ \\
\hline \multirow{2}{*}{1} & Canadian & Small & 1.91 & .1867 \\
& Canadian & Large & 1.92 & .2634 \\
2 & Israeli & Small & 1.57 & .6289 \\
& Israeli & Large & 1.88 & .5120 \\
3 & Israeli & Small & 1.29 & .6803 \\
& Israeli & Large & 1.54 & .5474 \\
\hline
\end{tabular}




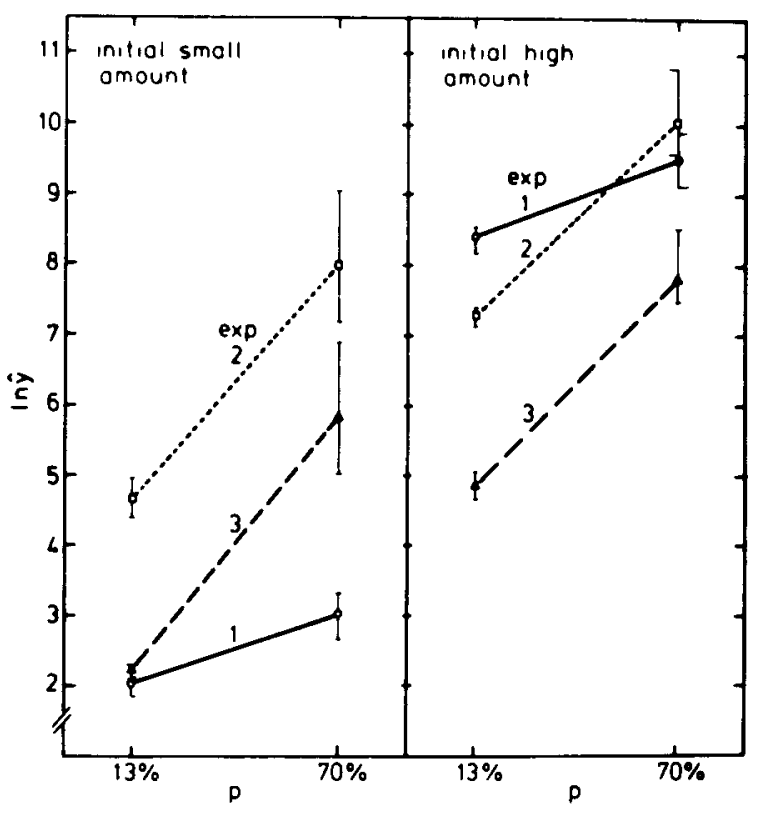

Figure 1. Median estimates and $95 \%$ confidence intervals (expressed in natural logarithms) as a function of inflation rate (P) and initial price for Experiments 1, 2, and 3.

which represents a significant improvement in accuracy, although, since $\beta$ does not reach the value of 1 , it leads to underestimation as well.

A similar conclusion can be reached from inspection of Figure 1, where $\ln \hat{Y}$ (ln of the median predicted price based on subjects' responses) is plotted against the corresponding rate of inflation $p$ and the $95 \%$ confidence intervals are indicated. The lines for the Israeli population are much steeper than those of the Canadian population, which represents the use of a larger exponent in each condition. Within each population, the lines for an initially small or large price are nearly parallel, indicating that the initial value did not affect accuracy or the corresponding $\beta \mathrm{s}$. This suggests that subjects from the same population used the same exponent independently of the condition to which they were assigned.

From the results and an inspection of Figure 1, it is clear that the Israeli subjects also exhibit underestimation of exponential growth (i.e., $\beta$ s smaller than 1, or in Figure 1 a slope smaller than 1). The important point, however, is that Israeli subjects give consistently better estimates and a more accurate perception of the power term, as reflected in the $\beta$ s.

The clear-cut difference in performance between the Israeli and Canadian subjects lends itself to several different interpretations. One plausible explanation may be that Israelis have had more experience using large numbers because of the pound unit (C\$1 = 60 pounds). Perhaps this experience alone (quite apart from inflation rate) would lead Israelis to be less timid about using large numbers when estimating exponential growth. A possibility to test this hypothesis emerged shortly after Experiment 2 was completed, when the Israeli unit of a "pound" was changed to a "shekel," the latter being equal to 10 pounds. If it is just getting used to large numbers that is responsible for the difference between Canadian and Israeli subjects, then Israelis using the new unit of a "shekel" should fall somewhere between the Canadians and the Israelis of Experiments 1 and 2.

\section{EXPERIMENT 3}

Experiment 3 was identical to Experiment 2, except that the numbers reflecting prices were divided by 10 and thus the prices were quoted in shekels. At the time the experiment was conducted, the official exchange rate between the Canadian dollar and the Israeli shekel was approximately 1:12.

\section{Results}

The results of Experiment 3 are summarized on the right-hand side of Table 1 , and they are highly similar to those of Experiment 2, as also are the estimated Bs (in Table 2). Figure 1 shows that the corresponding lines of Experiments 2 and 3 are close to parallel.

\section{GENERAL DISCUSSION}

The results of Experiment 3 refute the hypothesis that Israelis' estimates of exponential growth are better simply because they are used to dealing with larger numbers. Despite the use of different monetary units in Experiments 2 and 3, the resemblance between the results of these two experiments is indeed remarkable.

There are still several plausible explanations to account for the difference in performance of the two populations. One possibility is that the scale used by the Israeli subjects for exponential functions may differ from that of Canadian subjects. It is well known, from the scaling literature, that the apparent magnitude of numbers is not identical to their absolute value (e.g., Banks \& Hill, 1974), and that this relationship may depend on task parameters, among other things. For instance, Banks and Coleman (1981) have shown that "the apparent magnitude of numbers increases with a decelerated power function of their arithmetic magnitude when a series samples from an open-ended range," but "when an upper boundary of the range is specified, the subjective scale seems to be linear" (p. 15). Thus, Banks and Coleman (1981) argued for the existence of two subjective scales of numbers, depending on the nature of the task, namely whether an open- or a closed-ended range is used. Timmers and Wagenaar (1977), using the exponential growth paradigm, obtained similar results: They showed that underestimation for a de- 
scending (closed-range) series was negligible as compared with that for an ascending (open-range) series. It is thus possible that prolonged experience is just one of those task parameters which determine the nature of the subjective scale.

The more accurate estimation of Israeli subjects may also be explained as a by-product of the high inflation rate, which may force people to increase their awareness concerning all monetary matters; it could be argued that this is one of the dimensions on which the experience of Israeli and Canadian subjects differ. There is, however, no reason to believe that Israeli subjects had more experience on the particular task used in the three experiments.

Even if one accepts the conjecture that experience may play an important role in the estimation of exponential growth (or perhaps even more generally in the formation of subjective scales), the question still remains open as to what type of knowledge is acquired through experience. In particular, one may distinguish between experience that leads to the induction of coherent abstract principles and experience that leads only to the acquisition of limited, concrete information about specific tasks and/or situations (see also Caramazza, McLoskey, \& Green, 1981). If the former hypothesis is true, one would expect that experience in one task should be transferable to another task. For instance, one could suggest that Israelis might yield higher exponents than Canadians for other magnitude estimation tasks. We seriously doubt such a conjecture and would like to suggest that the role of experience is probably limited to the particular task being used.

In a certain respect, the findings reported in this paper are congruent with those reported by Hogarth (Note 1). Hogarth found that continuous feedback to his subjects improved their estimates. Similarly, we argue that subjects in the present experiments received feedback from the natural environment, and that this feedback was crucial in determining their performance. At any rate, for both Hogarth's subjects and ours, the question of "what exactly is learned by experience" remains open and requires further research.

\section{REFERENCE NOTE}

1. Hogarth, R. M. Functional and dysfunctional aspects of judgmental heuristics: A dynamic perspective (technical report). Chicago: University of Chicago, Center for Decision Research, Graduate School of Business, 1979.

\section{REFERENCES}

Banks, W. P., \& Coleman, M. J. Two subjective scales of number. Perception \& Psychophysics, 1981, 29, 95-105.

Banks, W. P., \& HiLl, D. K. The apparent magnitude of number scaled by random production. Journal of Experimental Psychology, 1974, 102, 353-376.

Caramazza, A., Mcloskey, M., \& Green, B. Naive beliefs in "sophisticated" subjects: Misconceptions about trajectories of objects. Cognition, 1981, 9, 117-123.

Luce, R. D. What sort of measurement is psychophysical measurement? American Psychologist, 1972, 27, 96-106.

Timmers, H., \& Wagenaar, W. A. Inverse statistics and misperception of exponential growth. Perception \& Psychophysics, $1977,21,558-562$.

TVensky, A., \& Kahneman, D. Judgment under uncertainty: Heuristics and biases. Science, 1974, 185, 1124-1131.

Wagenaar, W. A., \& Sagaria, S. Misperception of exponential growth. Perception \& Psychophysics, 1975, 18, 416-422.

WagenaAr, W. A., \& Timmers, H. Extrapolation of exponential time series is not enhanced by having more data points. Perception \& Psychophysics, 1978, 24, 182-184.

Wagenan, W. A., \& Trmmers, H. The pond-and-duckweed problem: Three experiments on the misperception of exponential growth. Acta Psychologica, 1979, 43, 239-251.

\section{NOTES}

1. In the static condition, the results of Wagenaar and his collaborators were replicated.

2. It should be emphasized here that the change is gradual, by which we mean that the parameters of a given heuristic or strategy may be more or less fixed for short periods, but change over longer periods, if required.

3. Note that, in contrast to previous studies, the exact rate of growth was given to the subject directly and did not have to be inferred. That change did not improve the subjects' estimates, as can be seen from the data in Tables 1 and 2 .

(Manuscript received July 16, 1981; revision accepted for publication May 6, 1983.) 